\title{
Références bibliographiques du dossier «Que savent les élèves? »
}

Cécile de Bouttemont et Sophie Condat

\section{(2) OpenEdition \\ 12 Journals}

Édition électronique

URL : http://journals.openedition.org/ries/244

DOI : 10.4000/ries.244

ISSN : 2261-4265

Éditeur

Centre international d'études pédagogiques

Édition imprimée

Date de publication : 1 décembre 2006

ISSN : 1254-4590

Référence électronique

Cécile de Bouttemont et Sophie Condat, «Références bibliographiques du dossier « Que savent les élèves? » », Revue internationale d'éducation de Sèvres [En ligne], 43 | décembre 2006, mis en ligne le 23 juin 2011, consulté le 03 mai 2019. URL : http://journals.openedition.org/ries/244 ; DOI : 10.4000/ ries. 244

(c) Tous droits réservés 


\title{
Références bibliographiques
}

\section{Cécile de Bouttemont Sophie Condat}

Cette bibliographie, non exhaustive, concerne en majorité des travaux produits ces dix dernières années. Elle a principalement pour cadre la scolarité du primaire au secondaire et se compose de deux parties: l'élaboration des contenus d'enseignement, et l'évaluation des acquis des élèves dans une perspective de pilotage du système éducatif.

La première partie rassemble des références sur les contenus d'enseignement et le rapport au savoir qu'ils impliquent. Plusieurs ouvrages analysent tout d'abord les enjeux sous-jacents à l'élaboration de tout programme d'enseignement; ils sont suivis de documents examinant les notions de savoir et de compétence. Des études internationales ou nationales sur l'élaboration des programmes et des curricula sont ensuite présentées. Les programmes d'enseignement en France et les débats actuels sur le socle de connaissances font l'objet d'un traitement séparé.

La seconde partie recense des documents concernant l'évaluation des acquis des élèves. Distinguant tout d'abord des documents de réflexion sur les évaluations des acquis des élèves, leurs finalités et leurs enjeux, elle s'intéresse ensuite aux évaluations menées à un niveau international puis au cas spécifique de la France. Enfin, quelques sites Internet offrant des ressources sur les évaluations internationales (PISA, PIRLS et TIMSS) et les évaluations nationales françaises sont signalés.

La majorité des documents mentionnés sont consultables au centre de ressources du Centre international d'études pédagogiques ou sont en ligne sur Internet.

Bibliographie arrêtée le 25 octobre 2006.

\section{Contenus d'enseignement et socle de connaissances}

\section{Définitions et enjeux}

\begin{abstract}
AUDIGIER François, CRAHAY Marcel, DOLZ Joaquim, Curriculum, enseignement et pilotage, De Boeck : Bruxelles, 2006, 276 p.

Que faut-il enseigner, comment l'enseigner et comment organiser la progression des apprentissages? Cet ouvrage réunit un ensemble de réflexions théoriques et d'études empiriques d'auteurs issus de champs disciplinaires différents. L'introduction s'attache à définir le champ conceptuel de la notion de curriculum dans les sciences de l'éducation. Les premières contributions analysent le curriculum dans une perspective sociologique et historique. Celles de la deuxième partie portent sur les mouvements entre le curriculum formel et le curriculum effectif. Enfin, la troisième partie s'intéresse aux relations entre les évaluations et les curricula officiels.
\end{abstract}




\section{DEMEUSE Marc, STRAUVEN Christiane, Développer un curriculum d'enseigne-}

ment ou de formation: des options politiques au pilotage, De Boeck/ Bruxelles, 2006, $301 p$.

Cet ouvrage retrace la démarche générale et les opérations particulières conduisant à la mise au point d'un curriculum. Dans une première partie sur les décisions préalables au développement d'un curriculum, les auteus mettent en avant l'importance du cadre philosophique et politique de l'éducation ainsi que ses finalités.

\section{DEPOVER Christian, NOEL Bernadette, Le curriculum et ses logiques: une approche contextualisée pour analyser les réformes et les politiques éducatives, L'Harmattan/Paris, 2005, 197 p.}

L'ouvrage met en lumière la place centrale qu'occupe le curriculum dans toute réforme du système éducatif à travers une approche systémique de l'enseignement de base, du secondaire, de l'université et de la formation en entreprise. Après un chapitre consacré à la définition du curriculum, les auteurs se penchent sur les éléments pouvant avoir une incidence sur la conception et la mise en oeuvre d'un tel outil, l'analyse de différents aspects des politiques, l'articulation entre les disciplines, enfin la complémentarité entre objectifs et compétences.

\section{FIGARI Gérard, GRANGEAT Michel, GAUTIER Gildas et al., L'évaluation du} curriculum : numéro thématique, Mesure et évaluation en éducation, septembre 2003, vol. 26, $n^{\circ} 3,83$ p., bibliogr.

Le curriculum est au centre des préoccupations de toutes les réformes éducatives. Il est devenu 160 l'un des objets d'étude les plus importants en sciences de l'éducation. De nombreuses questions sur les contenus, les modalités et les dispositifs d'enseignement voient le jour. Les quatre articles de ce numéro veulent contribuer à montrer l'intérêt des études sur l'évaluation du curriculum.

\section{GAUTHIER Roger-François, Les contenus de l'enseignement secondaire dans le monde : état des lieux et choix stratégiques, Unesco, 2006, 136 p. bibliogr.}

L'auteur, en s'appuyant sur les travaux d'organisations internationales telles que l'UNESCO, le BIE, l'OCDE ou le Conseil de l'Europe, offre un panorama analytique et synthétique de la question des contenus de l'enseignement secondaire dans le monde, des réformes mises en place et présente les tendances internationales et les principaux enjeux. Il montre en quoi les questions relatives aux contenus, longtemps délaissées, sont stratégiques pour les politiques éducatives. L'ouvrage a également pour finalité d'offrir une base solide de réflexion et de dégager un certain nombre d'orientations et de directives concrètes utiles pour toute réforme de contenus.

\section{FOURNIER Martine coord., Les mutations de l'école : le regard des sociologues,} Sciences humaines/Paris, 2006, 274 p.

Parmi un ensemble d'analyses, plusieurs contributions s'articulent autour de la question de la transmission des savoirs et des valeurs. Un entretien avec Bernard Charlot fait le point sur la dissociation que font les jeunes de lycées professionnels entre apprentissage scolaire et apprentissage de la vie. Un texte de Jean-Louis Derouet propose un aperçu historique des critiques auxquelles la réflexion sur les savoirs scolaires doit faire face. Deux contributions de Martine Fournier abordent la remise en question actuelle du découpage de l'enseignement en disciplines et les polémiques suscitées par les réformes des programmes scolaires. 
ISAMBERT-JAMATI Viviane, Les savoirs scolaires: enjeux sociaux des contenus d'enseignement et de leurs réformes, Paris: Editions universitaires, 1990, 233 p. L'auteur propose une réflexion sur les transformations des contenus d'enseignement dans une perspective historique et met en perspective la notion même de savoir. Les études présentées ici tentent de saisir la finalité des contenus scolaires et les fins poursuivies par ceux qui les mettent en œuvre.

LOMBARD Jean coord., L'école et les savoirs, L'Harmattan/Paris, 2001, 156 p. Qu'est-ce qu'apprendre? Cinq auteurs examinent sous des angles différents mais complémentaires la problématique de l'avenir de l'école. Ils mènent une analyse critique des relations entre l'école et les savoirs, à l'heure où se profile une logique libérale des apprentissages. Selon eux, en minimisant la transmission des savoirs et les compétences littéraires ou scientifiques, l'école s'appauvrit elle-même.

\section{LEWY Arieh, L'élaboration des programmes scolaires à l'échelon central et à} l'échelon des écoles, IIPE/Paris, 1992, 131 p.

Les programmes scolaires doivent-ils être conçus à l'échelon national ou par l'école? Doit-on associer les deux méthodes? L'auteur résume l'expérience des vingt dernières années et le rôle que les centres d'élaboration des programmes jouent. Il donne un aperçu historique des méthodes employées dans les différents pays du monde, montre les changements intervenus depuis la naissance du Mouvement pour la réforme des programmes scolaires (SBCD) et en évalue l'action.

\section{MARSH Colin, Key Concepts for Understanding Curriculum, Routledgefalmer/ New York, 2006, 274 p.}

Les curricula doivent équilibrer théorie et positionnements pratiques. Les vingt et un concepts clés retenus par l'auteur s'organisent en cinq chapitres : planification et développement du curriculum, gestion, perspectives pédagogiques, engagement collaboratif, idéologie. Des exemples concrets empruntés au monde anglo-saxon étayent ce guide.

\section{ROBERT André, Les contenus d'enseignement en question : histoire et actualité, CRDP de Bretagne/Rennes, 2000, 158 p.}

Cet ouvrage rassemble les différentes contributions issues du colloque qui s'est tenu à SaintBrieuc en mai 1999 sur «les contenus d'enseignement : histoire actualité». Parmi les thématiques abordées figurent la continuité de l'enseignement et l'articulation des disciplines. Un autre thème pose la question de savoir qui doit décider des contenus et de la place de partenaires extérieurs à l'éducation dans la rédaction des programmes.

\section{Savoirs et compétences dans les curricula}

Commission européenne, Compétences clés : un concept en développement dans l'enseignement général obligatoire, Direction générale de l'éducation et de la culture, Eurydice: Bruxelles, 2002, 188 p., téléchargées du site http://oraprod.eurydice.org

La notion de compétences clés, auparavant réservée à la formation professionnelle, suscite l'intérêt des responsables de la politique de l'éducation. Eurydice a réalisé une enquête sur la définition et l'intégration des compétences clés dans les programmes de l'enseignement général obligatoire de chaque pays de l'Union européenne. Après avoir rappelé les enjeux éducatifs européens et la définition et identification des compétences clés, l'étude se compose des rapports nationaux rappelant pour chacun des pays, la définition du concept de compétences clés, leur intégration dans les programmes d'enseignement et la manière dont elles sont évaluées. 
CRAHAY Marcel, Dangers, incertitudes et incomplétude de la logique de la compétence en éducation, Revue française de pédagogie, mars 2006, $n^{\circ} 154$, p. $97-110$

L'école est désormais le siège d'une nouvelle doxa : la pédagogie par compétence. L'auteur pose un regard critique sur cette idéologie pédagogique dominante dont l'origine est externe aux sciences de l'éducation. Pour lui, la définition même du concept de compétence est problématique et il suggère d'oublier la notion de compétence pour repenser celle de l'apprentissage.

\section{JONNAERT Philippe, Compétences et socioconstructivisme: un cadre théo- rique, De Boeck/Bruxelles, 2002}

Dans le contexte d'un vaste mouvement de réforme curriculaire, l'auteur identifie deux concepts à la base de ces réformes et tente de les analyser. Il montre ainsi la compatibilité des notions de compétence et de socioconstructivisme tout en les incluant dans un cadre théorique argumenté.

PERRENOUD Philippe, "Enseigner des savoirs ou développer des compétences : l'école entre deux paradigmes", in BENTOLILA Alain dir. Savoirs et savoir-faire, Nathan: Paris, 1995, bibliogr., p. 73-88

L'auteur de cette contribution analyse les notions de savoir et de compétence à l'école en soulignant les enjeux sociaux de l'une et l'autre et les rapports que ces deux notions entretiennent au sein du curriculum.

PERRENOUD Philippe, Construire des compétences dès l'école, ESF: Paris, 1998, 125 p., bibliogr.

De plus en plus, l'école se trouve devant ce dilemme : doit-elle transmettre des connaissances ou développer des compétences? L'auteur développe d'abord la notion de compétence, puis examine la question de la formation de compétences dans la scolarité générale, définit les implications pour le métier d'enseignant, enfin s'interroge pour savoir si cette question est un effet de mode ou une réponse décisive à l'échec scolaire.

\section{REY Bernard, CARETTE Vincent, Les compétences à l'école: apprentissage et évaluation, De Boeck/Bruxelles, 2003, 159 p.}

Cet ouvrage a pour but de fournir aux enseignants des outils pour faire acquérir et évaluer les compétences actuellement demandées dans les curricula scolaires de nombreux pays. La notion de compétence est abordée dans une première partie ainsi que le débat sur la légitimité et le bien-fondé des curricula par compétences. La deuxième partie traite de l'évaluation et propose un modèle d'évaluation des compétences en trois phases qui permet de repérer les difficultés éprouvées par chaque élève. Enfin, la troisième partie présente les résultats quantitatifs d'une enquête qui confirme la distinction entre trois degrés de compétences.

\section{ROPE Françoise, Savoirs et compétences: de l'usage de ces notions dans l'école et l'entreprise, L'Harmattan/Paris, 2004, 243 p.}

Le terme "compétence » tend aujourd'hui à s'imposer dans les discours tenus sur l'école ou sur les entreprises. Sociologues de l'éducation et du travail, didacticiens, tentent ici d'identifier le sens des changements qui s'opèrent en matière d'élaboration des contenus d'enseignement mais aussi de codification du travail dans les entreprises. 


\section{Études de cas}

BOSMAN C., GERARD F.M., ROEGIERS X., Quel avenir pour les compétences? De Boeck/Bruxelles, 2000

Avec le souci de mettre en évidence les convergences et divergences de l'approche par compétence appliquée dans la formation et les pratiques pédagogiques, cet ouvrage réunit des contributions situant l'approche par compétence dans ces deux mondes. Le chapitre trois sur «les compétences dans l'enseignement» cible plus particulièrement le programme de compétences en Belgique et Tunisie et pose la question de leur élaboration et des enjeux sous-jacents.

\section{CHARLOT Bernard dir., Les jeunes et le savoir : perspectives internationales,} Economica : Paris, 2001, 168 p.

Les travaux de l'auteur sur le rapport des jeunes avec le savoir s'élargissent ici dans une perspective internationale. Cependant, il ne s'agit pas, d'une recherche comparative stricto sensu mais d'une confrontation entre les sens conférés à la notion de rapport au savoir dans des aires culturelles diverses, en l'occurrence au Brésil, en France, en République tchèque et en Tunisie.

\section{CARPENTIER Claude, Contenus d'enseignement dans un monde en mutation :} permanences et ruptures, L'Harmattan : Paris, 2001, 382 p.

L'auteur analyse l'impact des transformations de la société sur l'enseignement, les savoirs transmis par l'école et la façon dont on enseigne. L'une des parties de l'ouvrage rassemble des textes sur le contexte politique et économique et ses conséquences sur les politiques éducatives et les contenus d'enseignement en Afrique australe, Afrique du Sud et CEI.

\section{COULBY David, Beyond the National Curriculum : curricular centralism and cultural diversity in Europe and the USA, Falmer Press/Londres, 2000, 132 p. \\ Cet ouvrage est consacré aux contenus d'enseignement aux États-Unis et en Europe. L'auteur souligne le paradoxe de l'hétérogénéité croissante de la société qui s'accompagne d'une centra- lisation des programmes. Il évalue le rôle joué par l'éducation à l'école et à l'université, remet- tant en question la nature des liens unissant l'État, l'économie et la connaissance.}

GREGOIRE Réginald, Le renouvellement du curriculum: expériences américaine, suisse et québécoise, Conseil supérieur de l'éducation, 1999, 111 p. téléchargées $d u$ site http://www.cse.gouv.qc.ca/pdfs/ren_cur3.pdf (consultation le 2 avril 2002)

Cette étude sur le curriculum fait partie d'un ensemble intitulé Le renouvellement $d u$ curriculum : expérience américaine suisse et québécoise. Dans une première partie, les auteurs présentent un concept issu des sciences de l'éducation américaines : l'éducation centrée sur le résultat (Outcome-Based Education] en le situant par rapport aux autres approches majeures issues du même courant général de pensée. La deuxième partie a pour objet la rénovation du curriculum québécois à travers quatre aspects : le cadre général de référence, le genre de curriculum et les ressources et stratégies pédagogiques.

\section{LE METAIS Joanna, International Trends in Primary Education: INCA} Thematic Study $N^{\circ}$ 9, Qualifications and Curriculum Authority: Londres, juin 2003, 128 p. téléchargées du site www.inca.org.uk

L'auteur rend compte des tendances internationales en matière d'éducation primaire à partir d'études menées ces vingt dernières années sur l'éducation primaire et en particulier les curricula dans dix-huit pays ou régions: Australie, Canada, Angleterre, France, Allemagne, Hongrie, Irlande, Italie, Japon, Corée, Pays-Bas, Nouvelle-Zelande, Singapour, Espagne, Suède, Suisse, États-Unis et Pays de Galles. 


\section{MOYLES Janet éd., HARGREAVES Linda éd, The primary curriculum : learning}

from international perspectives, Routledge et Kegan : Londres, 1998, 240 p.

Ces différents contributeurs analysent les relations et tensions en éducation entre les désirs des enfants et les demandes de la société auxquels les instituteurs doivent faire face quotidiennement. L'interaction entre enfance, programmes d'enseignement et pratiques de classse est ici placée dans une perspective internationale. La première partie présente un cadre de réflexion sur les programmes du primaire, la seconde partie, des études sur les programmes d'Afrique du sud, Australie, Asie du sud-est, Europe et États-Unis.

\section{ROEGIERS Xavier, DE KETELE Jean-Marie collab., Une pédagogie de l'intégra- tion: compétences et intégration des acquis dans l'enseignement, De Boeck Université : Bruxelles 2000, 304 p.}

Pourquoi développer des compétences dans l'enseignement et comment les développe-t-on? S'appuyant sur des recherches et des pratiques intégratrices dans les systèmes éducatifs, l'ouvrage traite la question du développement des compétences dans l'enseignement. La première partie propose un cadre de référence sur l'intégration et le développement des compétences d'un point de vue théorique et historique. La deuxième partie, pratique, détaille les implications de la pédagogie de l'intégration à quatre niveaux : élaboration des curriculums d'enseignement, pratiques de classe, modalités d'évaluation et manuels scolaires.

\section{RYCHEN D.S., TIANA A., Developping key competencies in education: some lessons from international and national experience, BIE/Genève, 2004, 80 p.}

164 Avec le soutien de l'OCDE, un programme international et interdisciplinaire sur les compétences a été lancé en 1997, le programme DeSeCo. Après une présentation du projet, des critères de sélection et des débats suscités, les auteurs décrivent en détail le référentiel des compétences clés. Celles-ci sont regroupées en trois catégories : capacité à agir de manière autonome - capacité à utiliser les outils que sont le langage, le savoir et les technologies de manière interactive - capacité à évoluer dans des groupes socialement hétérogènes.

\section{GAUTHIER Clermont, SAINT-JACQUES Diane, La réforme des programmes scolaires au Québec, Presses de l'Université de Laval/Québec, 2002, 225 p.}

Les textes rassemblés dans cet ouvrage ont été présentés lors d'un colloque de l'ACFAS (association canadienne-française pour l'avancement de la science) à l'Université de Montréal en 2000. Les auteurs situent la réforme actuelle de l'éducation au Québec à travers l'évolution des programmes scolaires depuis le milieu du XIX ${ }^{\mathrm{e}}$ siècle. La première partie de l'ouvrage est consacrée à l'étude du "Programme des programmes» avec une analyse approfondie de la notion de compétence. La deuxième partie traite, dans une perspective historique, des disciplines du nouveau «Programme de formation».

\section{LENOIR Y., LAROSE F., LESSARD C., Le curriculum de l'enseignement primaire : regards critiques sur ses fondements et ses lignes directrices, Editions du CRP/Sherbrooke, 2005, 421 p.}

Le nouveau curriculum de l'enseignement primaire a été progressivement mis en œuvre au Québec depuis septembre 2000. Il promeut une structuration et des contenus qui le différencient fortement du précédent curriculum, l'introduction de nombreux et profonds changements dans le processus d'enseignement-apprentissage. Les auteurs provenant des milieux universitaire et scolaire portent un regard distancié et critique sur ce document officiel. 


\section{France}

BIER Bernard dir., Savoirs de classe, savoirs de ville, savoirs de vie, VEI Enjeux, $n^{\circ} 123$, décembre 2000, $206 p$.

Dans ce dossier, les notions de savoirs, capacités et compétences sont clarifiées. Si l'école est un lieu où le rapport au savoir s'exerce, nombre de jeunes des milieux populaires lui opposent l'école de la vie, ce qui appelle des réponses différenciées, à la fois dans le cadre des enseignements scolaires, dans l'accompagnement périscolaire et dans les relations avec les familles. Par ailleurs, les migrants, les jeunes des quartiers développent des savoirs qui ne sont pas reconnus comme savoirs légitimes; les prendre en compte relève d'un acte politique autant que d'un pari scientifique.

\section{PERISSOL Pierre-André, Rapport d'information: La définition des savoirs} enseignés à l'école, Commission des affaires culturelles familiales et sociales, 13 avril 2005, 44 p. (consulté en septembre 2006), http://www.assemblee-nationale.fr/ 12/rap-info/i2247.asp

Quels savoirs enseigner, comment les enseigner? Pour l'auteur, les contenus et les finalités des savoirs enseignés sont un enjeu majeur. Dans ce rapport, il s'intéresse aussi aux performances des élèves français dans les enquêtes internationales ainsi qu'aux conditions d'une transmission réussie des connaissances et des compétences.

\section{DEMONQUE Chantal coord., Qu'est-ce qu'un programme d'enseignement?, Hachette Education, 1994, 172 p.}

Les contributions rassemblées apportent un certain nombre de réponses sur les programmes d'enseignement : comment et par qui les programmes sont-ils élaborés, à quels objectifs et impératifs cette élaboration est-elle subordonnée? Enfin, ces articles tentent de définir les liens entre les différents programmes existants et les savoirs de référence qui les inspirent.

École et collège: tout ce que nos enfants doivent savoir, XO Editions: Paris, 2006, 60 p.

La définition d'un socle commun a pour finalité de constituer une référence commune pour parents et enseignants. Il s'organise en sept compétences : cinq d'entre elles font l'objet des actuels programmes d'enseignement, deux autres ont été développées (les compétences sociales et civiques, l'autonomie et l'initiative des élèves). Chaque compétence est conçue comme une combinaison de connaissances fondamentales, de capacités à les mettre en œuvre dans des situations variées et d'attitudes.

"L'école en questions", Sciences humaines, octobre-novembre 2006, $n^{\circ}$ hors série, $n^{\circ} 5$.

Deux articles de ce numéro s'interrogent sur ce qui doit être enseigné. Dominique Raulin analyse le socle commun de connaissances et en souligne l'aspect décisif pour l'école en France alors que Philippe Meirieu le critique fortement considérant que les champs disciplinaires tels qu'ils sont enseignés devraient être repensés tout comme les moyens pédagogiques permettant de modifier le rapport au savoir des élèves les plus rétifs.

GAUTHIER Roger-François, Existe-t-il une exception française en matière de programme et d'enseignement? in La Revue de l'Inspection générale, septembre 2006, $n^{\circ} 3$, p.9-18.

Dans cet article, l'auteur s'interroge sur la gestion et la conception française des programmes d'enseignement. Le terme même de programme, spécifiquement français, est défini par rapport à celui de curriculum et de standard utilisés dans les autres pays. 
RAULIN Dominique, Les programmes scolaires : des disciplines souveraines au socle commun, Retz : Paris, 2006, 191 p.

Alors que le gouvernement souhaite rompre avec la logique disciplinaire et considérer la définition d'un socle commun de connaissances et de compétences pour tous les jeunes scolarisés, l'auteur fait une analyse des raisons de la remise en cause des programmes scolaires ces quinze dernières années. Il s'interroge sur les ressources disponibles pour faire évoluer les programmes mais aussi sur les contraintes. Il examine ensuite leur rôle de pilotage dans le système éducatif et la façon dont les enseignants les utilisent. Enfin, il aborde la problématique du socle commun en s'appuyant notamment sur l'expérience de différents pays européens.

\title{
VAN ZANTEN dir. Agnès, L'école, l'état des savoirs, Editions La Découverte/ Paris, 2000, 419 p.
}

Cet ouvrage collectif, rassemblant près d'une cinquantaine de contributions, présente un état des savoirs sur la situation de l'école française en privilégiant l'approche des sciences sociales. L'ensemble des thèmes a été regroupé en six rubriques. L'une d'elles, intitulée "Savoirs", présente des perspectives scientifiques complémentaires sur la sélection des savoirs enseignés et les savoirs appris, les pratiques pédagogiques et l'évaluation.

\section{Évaluation des acquis scolaires de l'élève et pilotage}

\author{
Cadre théorique : la notion \\ d'évaluation, les finalités \\ et les enjeux
}

BLAIS J.G., L'obligation de résultats à la lumière de l'interaction entre le qualitatif et la quantitatif; In LESSARD C., MEIRIEU P., L'obligation de résultats en éducation, De Boeck et Larcier, 2005, p. 123-144

Cette publication reprend les débats tenus lors des «Entretiens Jacques-Cartier» à l'université de Montréal en 2000. Les auteurs esquissent une réponse aux inquiétudes soulevées par l'obligation de résultats. Dans cet article plus particulièrement, l'auteur pose la question de la pertinence du résultat des évaluations.

BOTTANI Norberto coord., Les indicateurs comme outils des politiques éducatives, Politiques d'éducation et de formation. Analyses et comparaisons internationales, décembre 2001, $n^{\circ}$ 3, 126 p.

La qualité de l'éducation connaît une crise au milieu des années quatre-vingt, d'abord aux États-Unis avec la publication du rapport A Nation at Risk (1993), puis dans presque tous les pays de l'OCDE. C'est dans ce contexte qu'émerge l'idée de développer un programme d'indicateurs de l'enseignement, outils d'analyse et de pilotage indispensables pour améliorer la qualité de l'enseignement. Depuis 1992, l'OCDE publie Regards sur l'éducation. Les articles de ce numéro montrent la diversité de l'usage des indicateurs sur les politiques de l'éducation avec des analyses plus poussées concernant certains pays comme la France, les États-Unis ou l'Afrique francophone. 
BOTTANI Norberto coord., MAROY Christian, MONSEUR Christian et al., Évaluation des résultats scolaires des élèves: quels impacts sur les politiques d'éducation?, Politiques d'éducation et de formation. Analyses et comparaisons internationales, $n^{\circ} 11$, février 2004, p. 7-101

Les évaluations internationales des acquis des élèves tiennent aujourd'hui une place primordiale en politique de l'éducation. Leurs finalités et leurs enjeux, considérables, ne sont pas sans impact sur les politiques éducatives. Les auteurs de ce numéro portent un regard critique sur les limites de ces enquêtes auxquelles ils ont pris part.

DEMAILLY Lise, EMIN Jean-Claude, THELOT Claude et al., Évaluer, oui mais pour quoi faire? : dossier, Nouveaux regards, $n^{\circ}$ 16, mars 2002, p. 2-22.

Suite à la publication des résultats de PISA, l'Institut de recherches de la FSU (Fédération syndicale unitaire) a organisé un débat avec des spécialistes de l'évaluation. Ce débat constitue une part importante du dossier. Il est suivi d'un article sur les nouveaux enjeux de l'évaluation et de deux entretiens : l'un d'Agnès Van Zanten sur le bon usage de l'évaluation; l'autre de Claude Thélot sur les finalités de l'évaluation en éducation. La vraie question qui ressort de l'ensemble du dossier est celle de l'impact des résultats de PISA sur le pilotage des systèmes éducatifs et sur d'éventuelles réformes.

\section{PERRENOUD Philippe, L'évaluation des élèves: de la fabrication de l'excellence} à la régulation des apprentissages. Entre deux logiques, de Boeck Universitél Bruxelles/Belgique, 1998, 219 p., bibliogr. (Pédagogies en développement).

L'évaluation des élèves est partagée entre une logique certificative au service de la sélection et, depuis 1970, une logique formative au service des apprentissages. Celle-ci prend de plus en plus d'importance et remet en question le contrat pédagogique, la gestion de classe, la nature du curriculum et de l'excellence scolaire. À travers des textes déjà publiés ou inédits, avec pour fil rouge le rapport entre l'évaluation et la décision, l'ouvrage évoque l'oscillation de l'évaluation entre ces deux logiques coexistantes et insiste sur le fait que le véritable enjeu de l'évaluation est la lutte contre l'échec scolaire.

ROEGIERS Xavier, L'école et l'évaluation: des situations pour évaluer les compétences des élèves, de Boeck Université/Bruxelles, 2004, 367 p., bibliogr., index $\mathrm{Au}$ regard des enjeux politiques, philosophiques et éthiques qu'elle véhicule et de son impact sur les curricula, l'évaluation est l'une des questions centrales des systèmes éducatifs. L'ouvrage repense la question de l'évaluation des acquis des élèves : les quatre premiers chapitres cadrent la réflexion.

\section{Évaluations internationales}

\section{ADAMS Raymond coord., KIRSCH Irwin, DE LANGE Jan et al., Mesurer les connaissances et compétences des élèves: un nouveau cadre d'évaluation, OCDE/Paris, 1999, 91 .}

Les enquêtes internationales sont un outil de pilotage des systèmes d'enseignement qui doivent permettre de réorienter les politiques éducatives, de promouvoir les réformes de l'enseignement et donc d'améliorer la qualité de l'éducation. PISA, le programme international de l'OCDE pour le suivi des acquis des élèves, évalue tous les trois ans les savoirs et savoir-faire des jeunes de quinze ans dans les principaux pays industrialisés. L'ouvrage présente le cadre conceptuel sur lequel se fondent ces évaluations. Il décrit les domaines à évaluer (compréhension de l'écrit, culture mathématique et scientifique), l'objet des évaluations, la façon dont elles sont effectuées et leurs limites. 
CERI : Centre pour la recherche et l'innovation dans l'enseignement, L'évaluation formative: pour un meilleur apprentissage dans les classes secondaires, OCDE/ Paris, 2005, 306 p., bibliogr.

Cette étude de l'OCDE examine l'évaluation formative dans les établissements scolaires de huit pays : Angleterre, Australie, Canada, Danemark, Écosse, Finlande, Italie et NouvelleZélande. Elle dresse notamment un panorama des études réalisées au niveau international et analyse les politiques mises en œuvre. Elle montre comment l'évaluation formative peut être appliquée dans les classes et suggère comment les politiques peuvent promouvoir ce type d'approche pour améliorer l'enseignement.

CHINAPAH Vinayagum, L'éducation pour tous : quelle qualité? manuel pour le suivi permanent des acquis scolaires, UNESCO/Paris, 2000, 158 p., bibliogr., glossaire (L'éducation en devenir)

L'ouvrage donne un compte-rendu détaillé du projet UNESCO/UNICEF de suivi permanent des acquis scolaires dans cinq pays pilotes (Chine, Jordanie Mali, Maurice et Maroc); la présentation détaillée de quelques tests sur les acquis fondamentaux de l'apprentissage ainsi qu'un cadre d'action pour l'analyse des résultats des évaluations des élèves afin de promouvoir une éducation de qualité.

CROWNE Stephen, CYTERMANN Jean-Richard, KOCH Hans Konrad et al., Évaluation des connaissances et des compétences des élèves de 15 ans : questions et hypothèses formulées à partir de l'étude de l'OCDE - 31 mai 2002, ministère de la jeunesse, de l'Éducation et de la recherche. DESCO Direction de l'enseignement scolaire/Paris, 2002, 25 p. (Les rencontres de la DESCO).

168 Cette conférence-débat, qui se situe dans le cadre des Rencontres de la DESCO, est consacrée à l'évaluation des connaissances et des compétences des élèves de 15 ans. L'évaluation est-elle efficace dans la transmission des compétences et des connaissances des élèves? Quelles leçons tirer des résultats de l'enquête PISA? Comment faire en sorte que les évaluations soient plus utilisées pour modifier les pratiques d'enseignement? Des représentants de quatre pays européens (Grande-Bretagne, Italie, Allemagne, France) commentent les résultats de PISA et débattent autour de plusieurs points comme les modalités d'évolution possibles et l'évaluation des contenus d'enseignement.

DURU-BELLAT Marie, MONS Nathalie, SUCHAUT Bruno, Caractéristiques des systèmes éducatifs et compétences des jeunes de 15 ans: l'éclairage des comparaisons entre pays, IREDU/Dijon, janvier 2004, 23 p. téléchargées du site http://www.u-bourgogne.fr/IREDU

Prenant appui sur les données de PISA, cette étude de l'Institut de recherche sur l'éducation met en perspective les résultats des tests de compréhension de l'écrit de l'enquête avec certaines caractéristiques des systèmes éducatifs des pays participants. Son objectif est de comprendre ce qui influe sur le niveau des performances des élèves et d'expliquer la variabilité de l'ampleur des inégalités entre élèves et des inégalités sociales selon les pays.

OCDE : Organisation de coopération et de développement économiques, Cadre d'évaluation de PISA 2003 : connaissances et compétences en mathématiques, lecture, science et résolution de problèmes, OCDE/Paris, 2003, 226 p.

L'ambition de l'enquête PISA de l'OCDE est d'évaluer les performances des élèves par rapport à des objectifs définis certes par les systèmes éducatifs mais par la société. Après une présentation du cycle 2003 de PISA, le premier chapitre fait le point sur la culture mathématique, le deuxième sur la compréhension de l'écrit, le troisième sur la culture scientifique et le quatrième sur la résolution de problèmes. 
OCDE : Organisation de coopération et de développement économiques, What makes school systems perform? Seeing school systems through the prism of PISA, OCDE/Paris, 2004, 73 p., annexes, stat.

Quels sont les facteurs de réussite des systèmes éducatifs? En 2002, le ministère fédéral de l'éducation et de la recherche allemand lance une recherche afin de faire le lien entre les résultats de PISA 2000 et les principales politiques publiques de l'éducation. Le rapport présente les résultats de cette recherche pour le Canada, l'Angleterre, la Finlande, la France, les PaysBas et la Suède. Il s'inscrit dans le mouvement en cours qui met l'accent non plus sur les intrants des systèmes éducatifs mais sur les résultats de l'apprentissage et cherche à soutenir les pays pour améliorer la qualité de leur enseignement.

PEPPLER BARRY Ulrika coord., FISKE Edward B., Situation et tendances 2000 : l'évaluation des acquis scolaires, UNESCO/Paris, 2000, 72 p., annexes (Éducation pour tous).

Cette cinquième édition de "Éducation pour tous - Situation et tendances» s'intéresse à l'évaluation des acquis scolaires, aux principaux résultats des études et à la manière dont ces résultats peuvent améliorer l'enseignement et l'apprentissage.

POSTLETHWAITE Neville T., Le pilotage des résultats des élèves, Paris: UNESCO/Institut international de planification de l'éducation, 2005, 163 p. téléchargées du site http://www.unesco.org/iiep [consultation 22 mai 2006] (Principes de la planification de l'éducation).

De plus en plus de pays, surtout les pays développés, participent à des études sur le pilotage des résultats des élèves, démarche qui leur permet d'orienter leur politique éducative et de promouvoir des réformes susceptibles d'améliorer les performances de leur système éducatif. À l'aide d'exemples tirés d'études internationales, l'ouvrage explique notamment l'importance du pilotage des résultats des élèves, son évaluation et ses conséquences pour les responsables de l'éducation.

TIMSS performance assessment, Studies in educational evaluation, septembre 1999, vol. 25, $n^{\circ} 3$, p. 173-322.

Ce numéro spécial est consacré aux résultats internationaux des évaluations en sciences et en mathématiques (TIMSS : Third International Mathematics and Science Study).

\section{Afrique}

Association for the development of Education in Africa (ADEA), The Challenge of Learning : Improving the Quality of Basic Education in Sub Saharan Africa, 2003, 321 p. téléchargées du site http://www.adeanet.org

Le chapitre "pilotage de la performance : évaluation et examens» s'intéresse aux évaluations nationales et internationales, mais aussi à l'évaluation de la classe. On y étudie l'importance des évaluations, leur impact et leur utilisation dans le pilotage du système éducatif sudafricain.

CONFEMEN: Conférence des ministres de l'éducation des pays ayant le français en partage, L'éducation pour tous... tous pour l'éducation, CONFEMEN/ Dakar, OIF : Organisation internationale de la francophonie/Paris, 2006, cédérom En 1991, la CONFEMEN se dote d'un outil d'évaluation, le Programme PASEC (Plan d'Analyse des Systèmes Éducatifs des pays de la CONFEMEN). Ses objectifs sont l'identification de 
modèles d'écoles efficaces et peu coûteux, le développement dans chacun des États participants d'une capacité interne et permanente d'évaluation, la diffusion de méthodes, instruments et résultats en matière d'évaluation. Le cédérom contient notamment un dossier consacré au PASEC.

KELLAGHAN Thomas, GREANEY Vincent, Assessing student learning in Africa, The World Bank/Washington, 2004, 81 p., index, bibliogr.

Le programme "Éducation pour tous», soutenu par la communauté internationale, souligne l'importance de la qualité de l'apprentissage. L'ouvrage fait le point sur l'utilisation de l'évaluation pour piloter et évaluer l'apprentissage en Afrique sub-saharienne.

\section{Europe}

GAUTHIER Pierre-Louis coord., La généralisation de l'évaluation contribuet-elle à améliorer la qualité de l'enseignement?, Revue internationale d'éducation de Sèvres, décembre 2005, $n^{\circ}$ 40, p. 25-41.

L'évaluation s'est imposée progressivement en éducation. Elle fait désormais partie intégrante du système éducatif. Le dossier montre les limites de l'impact de l'évaluation sur l'amélioration de la qualité de l'enseignement. Les études de cas présentées ici concernent cinq pays : l'Espagne, l'Allemagne, le Royaume-Uni, la France et le Sénégal.

LE COQ Josette coord., LEVASSEUR Jacqueline coord., L'évaluation des élèves : dossier, Revue internationale d'éducation Sèvres, septembre 1996, n 11, p. 21-142, bibliogr.

170 Les articles de ce dossier évoquent, à travers les points de vue allemand, espagnol, français et britannique, mais aussi via les travaux de l'IEA (International Association for the Evaluation of Educational Achievement) et du Conseil de l'Europe, les différentes facettes de l'évaluation des élèves. Plusieurs contributions s'intéressent aux finalités de l'évaluation des acquis des élèves, pensée et utilisée comme outil de pilotage des curricula nationaux.

Reporting evaluation findings to policy makers, Réseau européen des responsables des politiques d'évaluation des systèmes éducatifs, mars 2004, 37 p., téléchargées de http://cisad.adc.education.fr/reva

Le but de l'étude est de donner une grille de lecture aux décideurs pour évaluer les résultats d'enquêtes internationales et préparer des travaux d'approfondissement au niveau national. Les conclusions sont basées sur un questionnaire de neuf questions basées sur les résultats de TIMSS et/ou PISA. Douze pays ont répondu : l'Autriche, la Belgique, l'Angleterre, la France, l'Allemagne, l'Irlande, le Luxembourg, les Pays-Bas, la Norvège, l'Ecosse, la Suède et l'Espagne. http://cisad.adc.education.fr/reva/pdf/ReportingEvaluation.pdf

\section{France}

BARDI Anne-Marie, BOUZAHER Myriem, DYCKMANS Annie et al., Les acquis des élèves, pierre de touche de la valeur de l'école?, ministère de l'Éducation nationale, de l'enseignement supérieur et de la recherche, juillet 2005, 85 p., rapport $n^{\circ}$ 2005-079 téléchargé $d u$ site http://lesrapports.ladocumentation francaise.fr

L'évaluation des acquis des élèves et le pilotage par les résultats doivent permettre d'améliorer l'efficacité du système éducatif. Le rapport porte sur cette question et sur l'évaluation des acquis des élèves, envisagée comme un référentiel à partir duquel interroger l'ensemble du système éducatif. http://lesrapports.ladocumentationfrancaise.fr/BRP/054000632/0000.pdf 
BOTTANI Norberto, VRIGNAUD Pierre, La France et les évaluations internationales, Haut Conseil de l'évaluation de l'école, janvier 2005, $n^{\circ}$ 16, 166 p. téléchargées de http://lesrapports.ladocumentationfrancaise.fr

Les enquêtes internationales sur l'évaluation des acquis des élèves permettent de comparer les résultats, de s'inspirer d'expériences éducatives, des cursus, des programmes d'enseignement d'autres pays. Le rapport s'intéresse notamment à l'historique des enquêtes internationales, à leur élaboration et leur mise en œuvre, leurs coûts et financements.

http://lesrapports.ladocumentationfrancaise.fr/BRP/054000359/0000.df

CEARD Marie-Thérèse, REMOND Martine, VARIER Michelle, L'appréciation des compétences des élèves et des jeunes en lecture et en écriture et l'évolution de ces compétences dans le temps : rapport établi à la demande du Haut Conseil de l'école, décembre 2003, 147 p. téléchargées du site: http://cisad.adc.education.fr/hcee (Évaluations françaises et internationales)

Ce rapport s'intéresse aux différentes formes de l'évaluation française, aux évaluations internationales et nationales et à leur impact sur les curricula.

IGEN: Inspection générale de l'Éducation nationale, IGAENR : Inspection générale de l'administration de l'Éducation nationale, Évaluation des enseignements et des pratiques enseignantes, évaluation des acquis des élèves IN Rapport annuel des inspections générales, Paris: Documentation française, 2005, p. 61-101

À quoi sert l'évaluation des acquis des élèves? Qui s'en sert? Pour quoi et comment? L'un des chapitres porte sur cette "question nouvelle pour de nouveaux enjeux». L'évaluation doit devenir consubstantielle au pilotage du système éducatif.

SUCHAUT Bruno, Les évaluations nationales en France outils pédagogiques et outils de pilotage, intérêts, limites et nouvelles perspectives, $19^{e}$ colloque de l'ADMEE EUROPE (11-13 septembre 2006, Luxembourg), Université de Bourgogne et IREDU C.N.R.S., Dijon, 17 p. téléchargées du site http://halshs.ccsd.cnrs.fr

L'auteur, chercheur en sciences de l'éducation, expose dans cette étude une analyse des épreuves des évaluations nationales de CE2 de français et de mathématiques et montre l'importance des outils d'évaluation de ce type à condition de ne pas se limiter à la seule approche institutionnelle. Il montre également comment les résultats des évaluations nationales des acquis des élèves pourraient influencer les curricula.

http://halshs.ccsd.cnrs.fr/docs/00/09/66/23/PDF/06035.pdf

BOUVIER Alain, THELOT Claude, TIANA FERRER Alejandro et al., Piloter par les résultats?, Administration et éducation, juin 2003, $n^{\circ}$ 98, p. 7-149

En France, la demande d'évaluation à l'école est très forte. Derrière le développement du pilotage par les résultats, pointe l'idée que le suivi des résultats de l'éducation constitue un instrument de progrès pour améliorer l'éducation. Les diverses contributions analysent les différents niveaux de pilotage par les résultats.

\section{Sitographie}

OCDE, PISA (Programme international pour le suivi des acquis des élèves), http://www.pisa.oecd.org

L'enquête PISA vise à mesurer et comparer les compétences des élèves de 15 ans dans l'ensemble des pays de l'OCDE. PISA 2000 portait principalement sur la compréhension de 
l'écrit, PISA 2003 sur la culture mathématique, PISA 2006 sera consacrée à la culture scientifique. Le site donne des informations sur ce programme de l'OCDE ainsi que les résultats de PISA 2000, PISA 2003 et PISA 2006 : les publications, les bases de données, les rapports nationaux, les rapports techniques.

IEA, PIRLS (Progress in International Reading Literacy Study), http://timss.bc. edu/pirls2001i/PIRLS2001_Pubs.html

En juin 2001, trente-cinq pays ont participé à l'enquête internationale PIRLS organisée par l'IEA (Association iternationale pour l'évaluation du rendement scolaire) en vue d'évaluer les performances en lecture des élèves achevant, en général, leur quatrième année de scolarité obligatoire. Les publications de PIRLS (rapport international, rapport technique, encyclopédie, la base de données, etc. ) sont en ligne sur le site.

\section{NCES, Trends in International Mathematics and Science Study (TIMSS), http://nces.ed.gov/timss}

Les données de TIMSS, programme international qui évalue les compétences et connaissances des élèves en mathématiques et en sciences, ont été collectées en 1995, 1999 et 2003. Les prochaines données paraîtront au printemps 2007. On trouvera sur le site des données sur le contexte de l'enquête, les résultats de TIMSS 1999 et 2003, les publications, les pays concernés et les partenaires.

Ministère de l'Éducation nationale, de l'enseignement supérieur et de la recherche, direction de l'évaluation et de la prospective (DEP), portail Éduc-Eval, http:// www.educ-eval.education.fr/evalint.htm

Conçu par la DEP, le portail Éduc-Éval présente les résultats de l'évaluation des compétences des élèves menée en France : évaluations diagnostiques, évaluations bilans du système éducatif, évaluations internationales comparatives.

Ministère de l'Éducation nationale, de l'enseignement supérieur et de la recherche, direction de l'évaluation et de la prospective (DEP), CISAD, le réseau européen des responsables des politiques d'évaluation des systèmes éducatifs, http://cisad. adc.education.fr/reva

Ce site propose ses études, ses projets et ses publications sur l'évaluation dans les systèmes éducatifs en Europe.

Désireux de favoriser l'émergence d'une culture européenne de l'évaluation et du pilotage des systèmes éducatifs, la Commission européenne et les États membres mènent depuis plusieurs années des actions de coopération, comme la mise en place du Réseau européen des responsables des politiques de l'évaluation des systèmes éducatifs. Le Réseau propose sur son site ses publications (lettre d'information, colloques et études).

Ministère de l'Éducation nationale, de l'enseignement supérieur et de la recherche, Évaluation et statistiques, http://www.education.gouv.fr/pid53/evaluation-etstatistiques.html

Ce site met en ligne les publications de la Direction de l'évaluation, de la prospective et de la performance: Repères et références statistiques; les notes [Information -Évaluation - Recherche]), les évaluations des rentrées de CE2 et de sixième ainsi que les indicateurs de résultats des lycées. 